\title{
Postprandial Lipoproteins and Cardiovascular Disease Risk in Diabetes Mellitus
}

\author{
Byambaa Enkhmaa • Zeynep Ozturk • \\ Erdembileg Anuurad • Lars Berglund
}

Published online: 27 January 2010

(C) The Author(s) 2010. This article is published with open access at Springerlink.com

\begin{abstract}
Diabetes mellitus is associated with increased risk for atherosclerotic cardiovascular disease (CVD). Recent prospective studies in healthy individuals suggest that the postprandial triglyceride (TG) level is a better independent predictor for assessing future CVD events than fasting TG levels. In contrast, results have been more controversial among diabetic patients, as some studies report a positive association between postprandial TG and CVD. This raises the issue of to what extent postprandial TG levels may be of predictive value in the diabetic population. One possibility impacting on the predictive power of postprandial TG in identifying CVD risk may be the presence of other risk factors, including alterations in lipid and lipoprotein metabolism, which could make it more difficult to identify the impact of postprandial lipemia on cardiovascular risk. The findings provide a challenge to develop a better approach to assess the impact of postprandial lipemia on CVD risk under diabetic conditions.
\end{abstract}

Keywords Diabetic dyslipidemia $\cdot$ Postprandial triglyceride - Cardiovascular risk - Diabetic and non diabetic population

\section{Introduction}

The prevalence of type 2 diabetes mellitus (DM) has grown rapidly in Westernized and developing countries, making it one of the most common diseases in the world. The number of adults with DM worldwide is estimated to increase from 135 million in 1995 to 300 million in 2025 [1]. This has a

B. Enkhmaa $\cdot$ Z. Ozturk $\cdot$ E. Anuurad $\cdot$ L. Berglund $(\bowtie)$ Department of Medicine, UCD Medical Center, CTSC, University of California, Davis,

2921 Stockton Boulevard, Suite 1400,

Sacramento, CA 95817, USA

e-mail: lars.berglund@ucdmc.ucdavis.edu significant negative health care potential, as DM is associated with a twofold to fourfold increased risk for development of atherosclerotic cardiovascular disease (CVD) [1], particularly coronary artery disease (CAD), and a threefold to eightfold greater risk for mortality from CVD. The high risk for CVD is underscored by the similar risks for a future CVD event in individuals with DM without a history of a prior myocardial infarction (MI), and in individuals without DM with a previous MI [2]. Moreover, more than $50 \%$ of all deaths among men or women with DM are attributable to CVD complications. These facts generally have been attributed to a high burden of risk factors such as hypertension, obesity, smoking, hyperglycemia, and dyslipidemia, many of which are associated with insulin resistance, resulting in a high cardiovascular risk phenotype among individuals with DM [3]. Among these risk factors, diabetic dyslipidemia is characterized by elevated levels of fasting triglyceride (TG); decreased levels of high-density lipoprotein (HDL) cholesterol; and presence of small, dense, low-density lipoprotein (LDL) cholesterol particles. It is noteworthy that TG and HDL cholesterol are powerful predictors of the postprandial response. In keeping with this, previous studies have shown an increased postprandial dyslipidemia in patients with DM regardless of elevated fasting TG concentrations [4, 5]. However, the potential role of postprandial conditions in the development of atherosclerosis is not well established in the diabetic population. Particularly, whereas recent studies have provided evidence for an association of elevated postprandial TG with an increased CVD risk in the general population, this issue has not been investigated to the same extent in the diabetic population.

\section{Postprandial Assessment: Methodological Aspects}

Accumulating evidence suggests that elevated levels of fasting and postprandial TG predict higher CVD risk [6, 7, 
8•, 9•]. The postprandial state is characterized by a rapid rise in triglyceride-rich lipoproteins (TRLs) derived from the intestine as chylomicrons, containing apolipoprotein (apo) B-48, and from the liver as very low density lipoprotein (VLDL), containing apo B-100. Although a close relationship exists between fasting and postprandial TG levels, intraindividual differences in TG levels between fasting and postprandial conditions vary substantially, partly due to differences in insulin sensitivity [10]. Although most studies of cardiovascular risk to date have focused on fasting conditions, recent studies have demonstrated that the postprandial TG level was a better independent predictor than the fasting $\mathrm{TG}$ level for assessing present and future CVD events in the nondiabetic population $[11 \bullet \cdot]$. Furthermore, increasing postprandial TG levels are more strongly and directly related to remnant lipoprotein concentrations than fasting TG levels [12••, 13]. This raises the possibility that risk for cardiovascular events may also be due to an increase in remnant lipoproteins in the postprandial state. The importance of postprandial TG in the prediction of CVD derives from the fact that in humans, most of the day is spent in this state. Studies that have monitored TG responses overnight following a fatcontaining evening meal have shown values to be elevated for 7-8 $\mathrm{h}$ after the meal, only falling toward fasting values between $4 \mathrm{AM}$ and $6 \mathrm{AM}$ [14].

Determination of the postprandial response itself is complex and goes beyond TG levels. Therefore, it has been challenging to assess the CVD risks associated with the postprandial state. Researchers have used several tools to investigate the postprandial lipemic responses, including responses to a metabolic challenge (fat tolerance or oral fatloading test) or a normal food intake, as well as use of selfdetermined diurnal capillary TG measurement $[9 \cdot, 12 \bullet, 15$, 16]. Typically, the oral fat load includes the administration of a test meal or drink with a high fat content $(\mathrm{eg}, 1 \mathrm{~g} /$ fat per kilogram of body weight) after an overnight fast. The increase in postprandial TG levels depends on the amount of dietary fat in the test meal; a very low $(5 \mathrm{~g})$ or low $(<15 \mathrm{~g})$ dose of dietary fat generally does not increase postprandial TG; moderate doses $(30-50 \mathrm{~g})$ dose dependently increase postprandial TG, whereas very high doses $(>80 \mathrm{~g})$ result in an exaggerated postprandial TG response [17-20]. In addition, the extent of postprandial lipemia is influenced by other dietary components, such as the content of protein [21•], fatty acid, glucose, or alcohol [22-24]. However, although metabolic ward testing (oral fat-loading test) may provide useful mechanistic information, it may not reflect regular, free-living daytime conditions. Interestingly, a relatively novel approach captures a daylong TG profile through serial capillary measurements $[25,26]$. In one study, participants were provided with a TG analyzer (TG-specific point-of-care testing device) to determine their own daytime TG profile at several time points on three different weekdays [26]. Thus, the daytime TG profile may have the potential to provide information regarding real-life TG changes and can be easily applied in clinical practice for routine screening of populations, reflecting in-hospital and out-of-hospital situations. Diurnal TG profiles have been associated with insulin sensitivity, fat mass, and diet [26]. Furthermore, the daytime TG profiles were closely related to postprandial lipemia as assessed by an oral fatloading test [27]. In a recent study, van Oostrom et al. [28•] compared different methods of investigating postprandial lipemia in 20 male participants with reduced insulin sensitivity and premature CAD. In their study, the TG load (daytime capillary TG measurement profile) test was more strongly associated with the parameters of postprandial lipemia than a more traditional oral fat-loading test.

\section{Postprandial Lipoproteins and CVD Risks in Nondiabetic Populations}

The associations of postprandial TG in response to normal food intake and cardiovascular events were the focus of several recent, large, prospective cohort studies. The Copenhagen City Heart Study [12••] investigated the role of postprandial TG in a nondiabetic population made up of 7587 women and 6394 men (20-93 years of age) observed over 26 years. After a fat tolerance test, plasma TG levels reached a mean peak level of $2.3 \mathrm{mmol} / \mathrm{L}$, whereas the mean peak level at $4 \mathrm{~h}$ after a normal food intake was $1.6 \mathrm{mmol} / \mathrm{L}$. After adjusting for other cardiovascular risk factors (eg, age, total cholesterol, body mass index, hypertension, diabetes, smoking, alcohol consumption, physical inactivity, lipid-lowering therapy, and postmenopausal status and hormone therapy in women), elevated postprandial TG levels were associated with increased risk of MI, ischemic heart disease, death [12••], and ischemic stroke in men and women [9•]. Another study by the same group of investigators confirmed these findings [8•]. Interestingly, the authors detected only minimal changes in the levels of lipids, lipoproteins, and apolipoproteins in response to normal food intake in the general population, which raises the issue of what might convey the increased postprandial CVD risk. Another large prospective study (the Women's Health Study) made up of 26,509 healthy US women older than 45 years of age (20,118 fasting; 6391 nonfasting participants) with 11.4 years of follow-up studied the association of fasting and postprandial TG levels and risk of future cardiovascular events [11••]. The authors found that higher postprandial TG levels were strongly associated with an increased risk of future events, independent of other risk factors (including HDL cholesterol and markers of insulin resistance). The hazard ratios 
for increasing tertiles of postprandial TG levels were as follows: 1.0 (reference), 1.44 (95\% CI, 0.90-2.29), and 1.98 (95\% CI, 1.21-3.25; $P=0.006$ for trend). Associations were strongest among individuals who had blood drawn $2-$ $4 \mathrm{~h}$ after a normal meal. Compared with the Copenhagen City Heart Study [12••], TG levels and event rate seemed to be considerably lower in the healthy US women. A recent study based on the Women's Health Study demonstrated that besides the TG level, postprandial HDL cholesterol, total/HDL cholesterol ratio, and apoA-1 levels predicted CVD [29••]. In contrast, postprandial total, LDL, and nonHDL cholesterol, in addition to apo B-100 levels and apo B-100/A-1 ratio, provided less useful CVD risk information [29••]. A significant association has been found between postprandial apo B-48 levels and the progression of CAD [30], and high levels of intestinally derived lipoproteins are associated with increased cardiovascular risk [31, 32]. Although the exact mechanisms remain to be established, previous studies have demonstrated that chylomicron remnants can enter the subendothelial space of the vascular wall with the potential to induce or participate in the development of atherosclerotic lesions [33, 34].

\section{Postprandial Lipoproteins in Type 2 Diabetes Mellitus}

Insulin resistance and DM are associated with multiple plasma lipid and lipoprotein abnormalities, including exaggerated postprandial lipemia, as well as increased CVD risk [35, 36]. Under normal, nondiabetic conditions, the postprandial plasma concentration of insulin is high, resulting in reduced endogenous VLDL production and reduced lipolysis from adipose tissue. However, in DM, the presence of insulin resistance leads to an increased capacity for VLDL-TG secretion and an increased residence time of TRL (Fig. 1). Insulin inhibits the production of large, buoyant $\mathrm{VLDL}_{1}$ particles (Svedberg's flotation unit, 60-400), and overproduction of these particles has been reported in patients with DM in the postprandial state $[37,38]$. In patients with DM, there is further increased production of apo B and reduced lipoprotein lipase activity, both of which can potentially promote a more pronounced postprandial response [39]. In addition, remnant lipoproteins, which are more likely to accumulate during conditions of partially reduced lipolysis, may be subject to reduced clearance through heparin sulfate proteoglycans under these conditions [40]. An increased postprandial residence time of TRL provides opportunities for lipid exchange of TG for cholesteryl esters between VLDL and LDL, mediated by cholesteryl ester transfer protein and resulting in TG enrichment of LDL particles and elevated formation of small, dense LDL [37]. In addition, there is an increased likelihood of changes in the HDL subfraction pattern in diabetes, with a reduction in the larger
HDL $2 b$ and an increase in the smaller HDL $3 b$ and $3 c$ particles [36].

\section{Postprandial Lipoproteins and CVD Risks in Diabetic Populations}

Contrary to the situation observed in the general population, only a few studies have examined the association between postprandial lipemia and CVD risk in diabetes, and the results have been inconclusive. The differential characteristics of study designs and participants in some studies are summarized in Table 1. Syvanne et al. [5] investigated 30 male participants with and 30 without CAD undergoing a fat-loading test $(760 \mathrm{kcal}, 78 \mathrm{~g}$ fat $=92.0$ energy percentage [E\%]); $50 \%$ of participants in each group had diabetes. In this study, postprandial TG levels were increased in both diabetic patient groups, with the most marked differences in the large $\mathrm{VLDL}_{1}$ fraction compared with control participants. TG levels in the two diabetic groups reached their peak levels $(\sim 4 \mathrm{mmol} / \mathrm{L})$ 4-6 h after the test meal. However, the authors did not find any association between postprandial TG and CAD events. A subsequent study from the same group of investigators, in which 43 (31 male, 12 female) participants with diabetes were studied, confirmed the initial overall finding [32]. No marked differences were found in the metabolism of intestinally or hepatically derived TRLs in patients with mild CAD $(<50 \%$ stenosis $)$ compared with those with severe CAD ( $>50 \%$ stenosis) after an oral fat load of a fatrich liquid formula ( $50 \mathrm{~g}$ fat $/ \mathrm{m}^{2}$ body surface). However, the authors observed greater postprandial excursions of apo B-48 and apo B-100 in intermediate-density lipoproteins in the participants with more severe CAD. Of note, there was a gender imbalance between the two groups, with more women in the mild coronary heart disease group compared with the severe coronary heart disease group (50\% vs $15 \%$ ).

In contrast, a study by Teno et al. [41] conducted among 61 (40 male, 21 female) patients with DM found an association between elevated levels of postprandial TG and increased carotid intima-media thickness (IMT) as assessed by ultrasonography. None of the study participants had clinical evidence of CVD or ischemic heart disease, and postprandial lipid levels were measured $4 \mathrm{~h}$ after a standard test meal that resembled a commonly consumed diet $(9 \mathrm{kcal} / \mathrm{kg} ; 20.0 \mathrm{E} \%$ fat). Notably, patients with normal fasting TG who developed postprandial hypertriglyceridemia had significantly greater carotid IMT than patients who remained normotriglyceridemic during the postprandial state. Postprandial plasma glucose, postprandial TG, and fasting LDL cholesterol levels were all independently correlated with carotid IMT; however, postprandial TG level showed the strongest correlation with the extent of carotid IMT $(P=0.002)$. The 


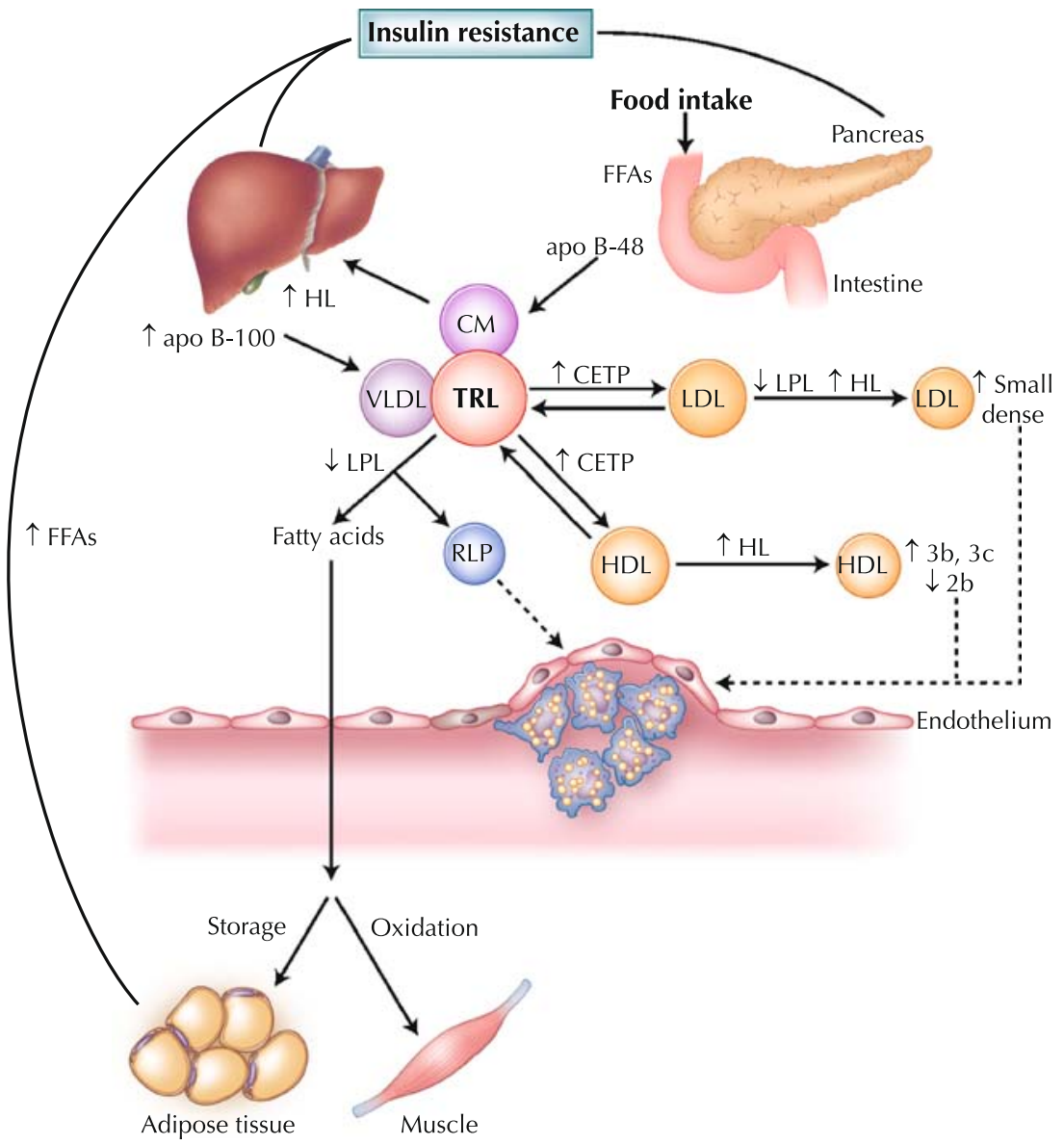

Fig. 1 Postprandial lipoprotein metabolism in diabetes. Insulin resistance plays a central role in the development of diabetic dyslipidemia. Under normal physiologic conditions, insulin suppresses lipolysis from adipose tissue and hepatic very low density lipoprotein $(V L D L)$ production. However, hyperinsulinemia in the postprandial state and insulin resistance in type 2 diabetes initiates a dyslipidemic triad of high triglyceride, low high-density lipoprotein $(H D L)$ cholesterol and high small, dense low-density lipoprotein $(L D L)$ levels. Prolonged residence of triglyceride-rich lipoproteins (TRLs)

findings of this study suggest that the role of postprandial hypertriglyceridemia in predicting CVD risks in the diabetic population may be more important in the early stages of atherosclerosis development.

Furthermore, a later study by Carstensen et al. [42] demonstrated that diabetic male patients with MI $(n=17)$ had a higher response of plasma TG and retinyl palmitateused as a marker of intestinal remnant lipoproteinscompared with diabetic male patients without MI $(n=15)$. Postprandial TG levels at $6(4.6 \mathrm{vs} 2.8 \mathrm{mmol} / \mathrm{L} ; P=0.04)$ and $8 \mathrm{~h}(3.6 \mathrm{vs} 2.4 \mathrm{mmol} / \mathrm{L} ; P=0.03)$, as well as the incremental area under the curve for the postprandial period (560 vs $297 \mathrm{mmol} \times 480 \mathrm{~min} / \mathrm{L} ; P=0.048$ ) after a fat-loading test $(1027 \mathrm{kcal}, 86.6 \mathrm{~g}$ fat $=77.0 \mathrm{E} \%)$ were increased in patients with MI compared with those in patients without MI. The authors suggested that the contradictory outcomes of their in the circulation promotes the transfer of $H D L$ or $L D L$ cholesteryl esters for triglyceride, mediated by cholesteryl ester transfer protein $(C E T P)$. LDL can undergo hydrolysis by hepatic lipase $(H L)$ or lipoprotein lipase $(L P L)$, which hydrolyzes triglycerides from the core of $L D L$, resulting in production of smaller, denser particles. Moreover, triglyceride-enriched $H D L$ particles become smaller, denser $(H D L 3 \mathrm{~b}$ and $3 \mathrm{c}$ ) and are more rapidly catabolized, contributing to low plasma $H D L$ in insulin resistance and type 2 diabetes. apo apolipoprotein; $C M$ chylomicron; FFA free fatty acid; $R L P$ remnant lipoprotein

study and those by Syvanne et al. [5] and Mero et al. [32] may be due to differences in 1) study populations, 2) severity of $\mathrm{CAD}, 3$ ) diets and meal testing, 4) medications, and 5) degree of matching of the control and case groups. The participants in the study by Carstensen et al. [42] were almost a decade older, had higher body mass index, and may have had more severe CAD (as they had verified MI). They were also fed a high-carbohydrate diet for 3 days before the fat-loading test to achieve minimal day-to-day variation in insulin sensitivity. In addition, there were differences in the treatment regimen, as $80 \%$ of diabetic patients with $\mathrm{CAD}$ consumed $\beta$ blockers, and several patients without CAD received metformin in the study by Syvanne et al. [5]. In the study by Carstensen et al. [42], the $\beta$-blocker treatment rate was $41 \%$, and metformin treatment was an exclusion criterion. A total of $50 \%$ of those in the mild CAD group 


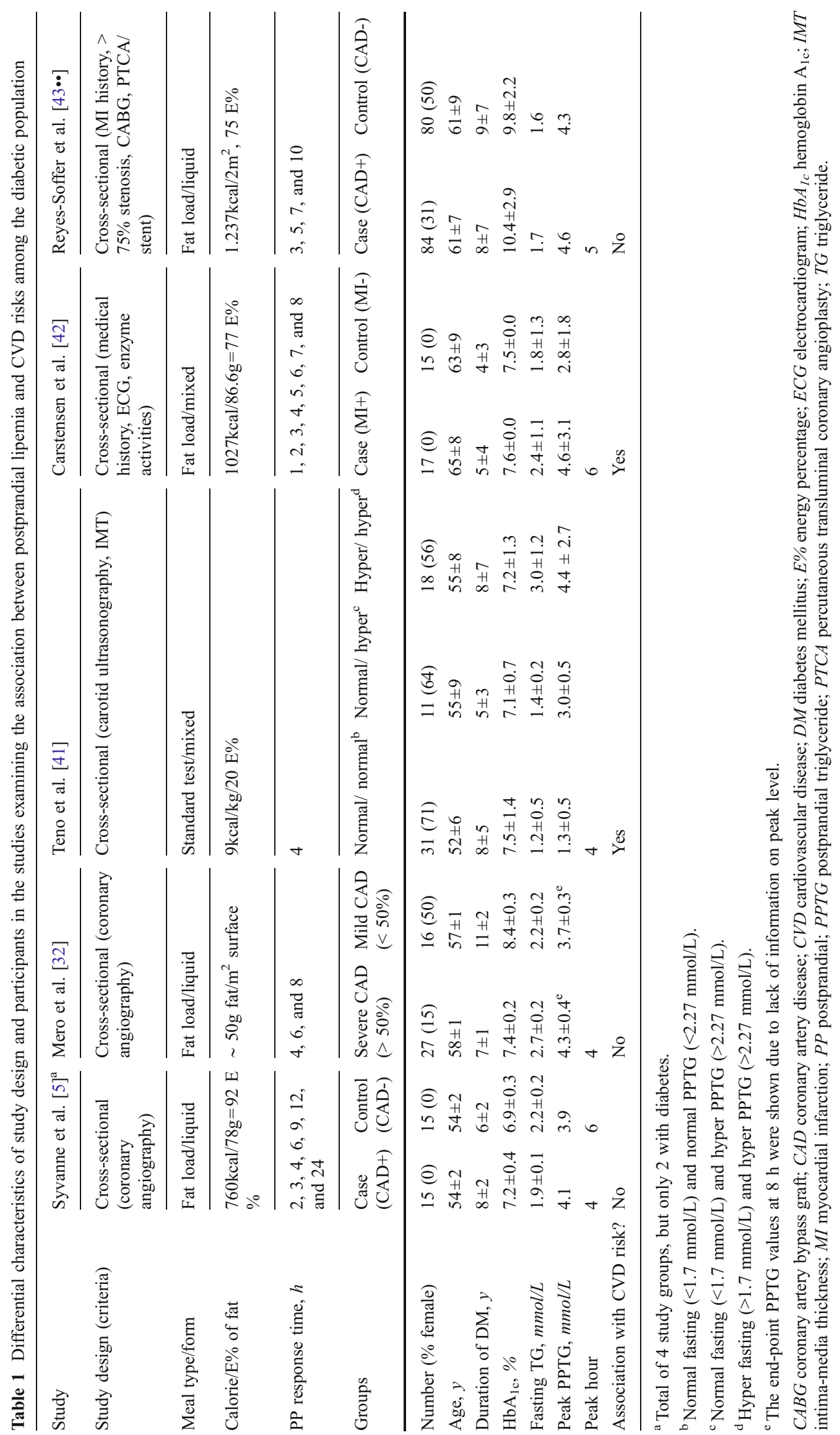


in the study by Mero et al. [32] received insulin, compared with $22 \%$ of the severe CAD group, whereas insulin therapy was an exclusion criterion in the study by Carstensen et al. [42]. In the Mero et al. [32] study, the glycemic control in the mild CAD group was significantly worse than that of the severe CAD group $(P=0.007)$; the duration of the diabetes was longer in the mild CAD group $(P=0.04)$; and the mild CAD group had a skewed gender distribution, with $50 \%$ female compared with nearly all male patients in the severe CAD group ( $P=0.013)$, all of which may impact the findings.

As previous studies have yielded inconsistent findings regarding the association between postprandial TG and CAD severity among diabetic populations, Reyes-Soffer et al. [43••] recently tested the hypothesis that more subtle differences (eg, size and number of TRLs) may be a better discriminator for case-control status under diabetic conditions. They conducted a cross-sectional, case-control study with 8.7 years of follow-up in diabetic individuals (84 with, 80 without CAD). It was hypothesized that diabetic patients with and without CAD would have similar postprandial TG levels, but patients with CAD would have a greater number of small postprandial lipoprotein particles (in the density range of $d<1.006$ [a mixture of chylomicrons, chylomicron remnants, and VLDL]). However, no significant association between the presence of CAD and any measures of postprandial lipoproteins (postprandial TG, $d<1.006 \mathrm{TG}$, postprandial apo B-48, total apo $\mathrm{B}, d<1.006$ particle number and size distributions, or remnant lipoprotein levels) after a high fat-loading test using a high-fat liquid formula $\left(1.237 \mathrm{kcal} / 2 \mathrm{~m}^{2}\right.$ body surface, $75.0 \mathrm{E} \%$ fat $)$ was noted. Gender-specific analysis revealed no casecontrol difference. Notably, there were no significant differences in postprandial lipoproteins at baseline between individuals without $\mathrm{CAD}$ at baseline who did not develop $(n=33)$ or who subsequently developed CAD $(n=36)$ and the original CAD group patients. A significant difference between the study by Carstensen et al. [42] and that by Reyes-Soffer et al. [43••] was a difference in the fasting TG level between cases and control participants. TG levels were $30 \%$ higher in cases than control participants (2.35 vs $1.80 \mathrm{mmol} / \mathrm{L}$ ) in the former study, whereas levels were similar in cases and control participants (1.63 vs $1.68 \mathrm{mmol} / \mathrm{L}$ ) in the latter study. Because fasting TG is a major determinant of postprandial TG levels [44], the differences in the fasting TG levels between cases and control participants in the study by Carstensen et al. [42] may have contributed to the observed differences in the postprandial TG excursions. Another difference was the longer duration of diabetes and higher hemoglobin $\mathrm{A}_{1 \mathrm{c}}\left(\mathrm{Hb}_{1 \mathrm{c}}\right)$ levels, indicating worse glycemic control in the latter study. However, although $\mathrm{HbA}_{1 \mathrm{c}}$ levels were considerably lower in the three previous postprandial studies in diabetic patients [5, 32, 42], two of them [5, 32] found essentially the same results as the study by Reyes-
Soffer et al. [43••]. This suggested that a difference in $\mathrm{HbA}_{1 \mathrm{c}}$ levels might not impact substantially on any association between postprandial TG and case-control status.

Elevated LDL levels are associated with increased CVD risk, and small, dense LDL particles, relatively more common in diabetes, have a high susceptibility to oxidation. Oxidative modification of LDL results in rapid uptake by macrophages, which leads to foam cell formation and promotion of the expression of intercellular adhesion molecule-1 [45]. Furthermore, LDL oxidation in the postprandial state seems to be affected by an acute increase in glycemia. Apo B in LDL can be modified by advanced glycosylation end products, reducing the binding affinity of the LDL-modified particles to their hepatic receptors and subsequently leading to increased oxidation of these particles [46]. In addition, glycated and oxidized LDL particles induce the release of foam cell cytokines (tumor necrosis factor- $\alpha$, interleukin-1), which play important roles in the atherosclerotic process $[47,48]$. Thus, oxidative modification of LDL may contribute to higher CVD risk among diabetic patients, and elevated levels of TG may contribute to the rapid LDL oxidation seen in DM.

Tentolouris et al. [49] demonstrated that normotriglyceridemic diabetic patients with microalbuminuria had threefold increased postprandial TG levels compared with diabetic patients without microalbuminuria after ingestion of a standard mixed test meal $(783 \mathrm{kcal}, 52.5 \mathrm{E} \%$ fat). Microalbuminuria is an established independent risk factor for CVD in patients with $\mathrm{DM}$, and its association with excessive postprandial lipemia may partially explain the increased CVD risk in this group of patients. Postprandial TG increase in DM patients with diabetic nephropathy was markedly higher than it was in those without diabetic nephropathy [50], and the fasting apo B-48 level was elevated in individuals with advanced diabetic nephropathy [51]. A later study by the same group of investigators showed that apo B-48 after a standard test meal $(460 \mathrm{kcal}, 18.0 \mathrm{~g}$ fat $=35.0 \mathrm{E} \%)$ was significantly higher in patients with diabetic nephropathy than in control participants and was significantly associated with an increase in small, dense LDL particles [52].

\section{Conclusions}

Overall, unlike the situation in the nondiabetic population, in which measurement of postprandial TG levels has been useful in identifying individuals at high risk for the presence of CVD and future CVD events, specifically testing for postprandial TG level has to date shown considerably less promise in patients with DM. One possibility is that the predictive power of measuring postprandial TG to identify CVD risk is diminished in diabetes due to an already altered lipid and lipoprotein metabolism. Furthermore, once diabetes develops, patients are exposed to a wide variety of other risk factors that 
place them at increased risk for developing CVD. In the United Kingdom Prospective Diabetes Study, fasting TG level was associated with CAD after adjusting for age and sex but was not an independent risk factor when other variables were included in the model [53]. Thus, the high level of CVD risks in patients with DM exposed to a high cardiovascular risk phenotype may make it more difficult to identify any independent contribution of individual risk factors. For example, obesity, decreased physical activity, and raised insulin concentrations provide an increased risk for CVD in the general population but have not been associated with risk in patients with DM [53]. These variables are also risk factors for diabetes, but once diabetes has developed, increased levels of LDL cholesterol, decreased levels of HDL cholesterol, presence of hypertension, microalbuminuria, and hyperglycemia are likely greater risk factors for CVD. To put this in simple terms, the risk contributed by postprandial lipemia may drown in the sea of risk factors in patients with DM. However, these findings do not suggest an absence of any association between postprandial lipemia and CVD risk in diabetic populations. Instead, the mixed message in the studies to date provides a challenge to develop more precise approaches or tools that could allow us to assess the association of postprandial TG levels and CVD risks in more detail. At present, further studies are needed to establish the clinical usefulness of measuring postprandial TG in individuals with DM as a tool to assess a high risk for CVD.

Disclosure This project was supported by grants 49735 (Pearson TA, principal investigator) and 62705 (Berglund L, principal investigator) from the National Heart, Lung, and Blood Institute. This work was supported in part by the UC Davis CTSC (RR 024146) and by a grant from the Nora Eccles Treadwell Foundation. No other potential conflicts of interest relevant to this article were reported.

Open Access This article is distributed under the terms of the Creative Commons Attribution Noncommercial License which permits any noncommercial use, distribution, and reproduction in any medium, provided the original author(s) and source are credited.

\section{References}

Papers of particular interest, published recently, have been highlighted as:

- Of importance

•- Of major importance

1. King H, Aubert RE, Herman WH: Global burden of diabetes, 1995-2025: prevalence, numerical estimates, and projections. Diabetes Care 1998, 21:1414-1431.

2. Haffner SM, Lehto S, Ronnemaa $\mathrm{T}$, et al.: Mortality from coronary heart disease in subjects with type 2 diabetes and in nondiabetic subjects with and without prior myocardial infarction. N Engl J Med 1998, 339:229-234.

3. Grundy SM, Benjamin IJ, Burke GL, et al.: Diabetes and cardiovascular disease: a statement for healthcare professionals from the American Heart Association. Circulation 1999, 100:1134-1146.

4. Rivellese AA, De Natale C, Di Marino L, et al.: Exogenous and endogenous postprandial lipid abnormalities in type 2 diabetic patients with optimal blood glucose control and optimal fasting triglyceride levels. J Clin Endocrinol Metab 2004, 89:2153-2159.

5. Syvanne M, Hilden H, Taskinen MR: Abnormal metabolism of postprandial lipoproteins in patients with non-insulindependent diabetes mellitus is not related to coronary artery disease. J Lipid Res 1994, 35:15-26.

6. Hokanson JE, Austin MA: Plasma triglyceride level is a risk factor for cardiovascular disease independent of high-density lipoprotein cholesterol level: a meta-analysis of populationbased prospective studies. $J$ Cardiovasc Risk 1996, 3:213-219.

7. Sarwar N, Danesh J, Eiriksdottir G, et al.: Triglycerides and the risk of coronary heart disease: 10,158 incident cases among $\mathbf{2 6 2 , 5 2 5}$ participants in 29 Western prospective studies. Circulation 2007, 115:450-458.

8. - Langsted A, Freiberg JJ, Nordestgaard BG: Fasting and nonfasting lipid levels: influence of normal food intake on lipids, lipoproteins, apolipoproteins, and cardiovascular risk prediction. Circulation 2008, 118:2047-2056. Lipid profiles at most change minimally in response to normal food intake in the general population, and postprandial lipid profiles predicted increased CVD risk (the Copenhagen General Population Study and Copenhagen City Heart Study).

9. • Freiberg JJ, Tybjaerg-Hansen A, Jensen JS, et al.: Nonfasting triglycerides and risk of ischemic stroke in the general population. JAMA 2008, 300:2142-2152. Elevated postprandial $T G$ level was associated with increased risk of ischemic stroke (Copenhagen City Heart Study).

10. Delawi D, Meijssen S, Castro Cabezas M: Intra-individual variations of fasting plasma lipids, apolipoproteins and postprandial lipemia in familial combined hyperlipidemia compared to controls. Clin Chim Acta 2003, 328:139-145.

11. • Bansal S, Buring JE, Rifai N, et al.: Fasting compared with nonfasting triglycerides and risk of cardiovascular events in women. JAMA 2007, 298:309-316. Postprandial TG level was a better independent predictor than fasting $T G$ level for assessing present and future CVD events in 26,509 healthy US women (Women's Health Study).

12. •- Nordestgaard BG, Benn M, Schnohr P, et al.: Nonfasting triglycerides and risk of myocardial infarction, ischemic heart disease, and death in men and women. JAMA 2007, 298:299 308. Elevated postprandial TG level was associated with increased risk of MI, ischemic heart disease, and death among 7587 women and 6394 men after adjusting for other CVD risk factors (Copenhagen City Heart Study).

13. Nakajima K, Nakano T, Moon HD, et al.: The correlation between TG vs remnant lipoproteins in the fasting and postprandial plasma of 23 volunteers. Clin Chim Acta 2009, 404:124-127.

14. Williams CM, Moore F, Morgan L, et al.: Effects of n-3 fatty acids on postprandial triacylglycerol and hormone concentrations in normal subjects. Br J Nutr 1992, 68:655-666.

15. Schaefer EJ, Audelin MC, McNamara JR, et al.: Comparison of fasting and postprandial plasma lipoproteins in subjects with and without coronary heart disease. Am J Cardiol 2001, 88:1129-1133.

16. Cohn JS, McNamara JR, Cohn SD, et al.: Postprandial plasma lipoprotein changes in human subjects of different ages. $J$ Lipid Res 1988, 29:469-479. 
17. Cohen JC, Noakes TD, Benade AJ: Serum triglyceride responses to fatty meals: effects of meal fat content. $\mathrm{Am} \mathrm{J}$ Clin Nutr 1988, 47:825-827.

18. Dubois C, Armand M, Azais-Braesco V, et al.: Effects of moderate amounts of emulsified dietary fat on postprandial lipemia and lipoproteins in normolipidemic adults. Am J Clin Nutr 1994, 60:374-382.

19. Murphy MC, Isherwood SG, Sethi S, et al.: Postprandial lipid and hormone responses to meals of varying fat contents: modulatory role of lipoprotein lipase? Eur J Clin Nutr 1995, 49:578-588.

20. Dubois C, Beaumier G, Juhel C, et al.: Effects of graded amounts $(0-50 \mathrm{~g})$ of dietary fat on postprandial lipemia and lipoproteins in normolipidemic adults. Am J Clin Nutr 1998, 67:31-38.

21. - Mortensen LS, Hartvigsen ML, Brader LJ, et al.: Differential effects of protein quality on postprandial lipemia in response to a fat-rich meal in type 2 diabetes: comparison of whey, casein, gluten, and cod protein. Am J Clin Nutr 2009, 90:41-48. Whey protein as a supplement to a fat-rich meal improved postprandial lipemia in patients with type $2 \mathrm{DM}$.

22. Tinker LF, Parks EJ, Behr SR, et al.: (n-3) fatty acid supplementation in moderately hypertriglyceridemic adults changes postprandial lipid and apolipoprotein $B$ responses to a standardized test meal. $J$ Nutr 1999, 129:1126-1134.

23. Cohen JC, Berger GM: Effects of glucose ingestion on postprandial lipemia and triglyceride clearance in humans. $J$ Lipid Res 1990, 31:597-602.

24. van Tol A, van der Gaag MS, Scheek LM, et al.: Changes in postprandial lipoproteins of low and high density caused by moderate alcohol consumption with dinner. Atherosclerosis 1998, 141(Suppl 1):S101-S103.

25. van Wijk JP, Cabezas MC, Halkes CJ, et al.: Effects of different nutrient intakes on daytime triacylglycerolemia in healthy, normolipemic, free-living men. Am J Clin Nutr 2001, 74:171-178.

26. van Oostrom AJ, Castro Cabezas M, Ribalta J, et al.: Diurnal triglyceride profiles in healthy normolipidemic male subjects are associated to insulin sensitivity, body composition and diet. Eur J Clin Invest 2000, 30:964-971.

27. Castro Cabezas M, Halkes CJ, Meijssen S, et al.: Diurnal triglyceride profiles: a novel approach to study triglyceride changes. Atherosclerosis 2001, 155:219-228.

28. - van Oostrom AJ, Alipour A, Sijmonsma TP, et al.: Comparison of different methods to investigate postprandial lipaemia. Neth J Med 2009, 67:13-20. The daytime capillary $T G$ profile was more strongly associated with parameters of postprandial lipemia compared with a more traditional oral fatloading test.

29. •• Mora S, Rifai N, Buring JE, et al.: Fasting compared with nonfasting lipids and apolipoproteins for predicting incident cardiovascular events. Circulation 2008, 118:993-1001. In addition to TG, postprandial HDL cholesterol, total/HDL cholesterol ratio, and apo A-1 levels predicted CVD; however, postprandial total, LDL, and non-HDL cholesterol; apo B-100 levels; and apo B-100/A-1 ratio provided less useful CVD risk information (Women's Health Study).

30. Karpe F, Steiner G, Uffelman K, et al.: Postprandial lipoproteins and progression of coronary atherosclerosis. Atherosclerosis 1994, 106:83-97.

31. McNamara JR, Shah PK, Nakajima K, et al.: Remnant-like particle (RLP) cholesterol is an independent cardiovascular disease risk factor in women: results from the Framingham Heart Study. Atherosclerosis 2001, 154:229-236.
32. Mero N, Malmstrom R, Steiner G, et al.: Postprandial metabolism of apolipoprotein B-48- and B-100-containing particles in type 2 diabetes mellitus: relations to angiographically verified severity of coronary artery disease. Atherosclerosis 2000, 150:167-177.

33. Proctor SD, Vine DF, Mamo JC: Arterial retention of apolipoprotein $\mathrm{B}(48)$ - and $\mathrm{B}(\mathbf{1 0 0})$-containing lipoproteins in atherogenesis. Curr Opin Lipidol 2002, 13:461-470.

34. Vine DF, Takechi R, Russell JC, et al.: Impaired postprandial apolipoprotein-B48 metabolism in the obese, insulin-resistant JCR:LA-cp rat: increased atherogenicity for the metabolic syndrome. Atherosclerosis 2007, 190:282-290.

35. Battula SB, Fitzsimons O, Moreno S, et al.: Postprandial apolipoprotein B48-and B100-containing lipoproteins in type 2 diabetes: do statins have a specific effect on triglyceride metabolism? Metabolism 2000, 49:1049-1054.

36. Krauss RM: Lipids and lipoproteins in patients with type 2 diabetes. Diabetes Care 2004, 27:1496-1504.

37. Pastromas S, Terzi AB, Tousoulis D, et al.: Postprandial lipemia: an under-recognized atherogenic factor in patients with diabetes mellitus. Int $J$ Cardiol 2008, 126:3-12.

38. Adiels M, Olofsson SO, Taskinen MR, et al.: Diabetic dyslipidaemia. Curr Opin Lipidol 2006, 17:238-246.

39. Ira J. Goldberg: Diabetic Dyslipidemia: Causes and Consequences. The Journal of Clinical Endocrinology \& Metabolism 2001, 86:965971.

40. Mahley RW, Ji ZS: Remnant lipoprotein metabolism: key pathways involving cell-surface heparan sulfate proteoglycans and apolipoprotein E. J Lipid Res 1999, 40:1-16.

41. Teno S, Uto Y, Nagashima H, et al.: Association of postprandial hypertriglyceridemia and carotid intima-media thickness in patients with type 2 diabetes. Diabetes Care 2000, 23:14011406.

42. Carstensen M, Thomsen C, Gotzsche O, et al.: Differential postprandial lipoprotein responses in type 2 diabetic men with and without clinical evidence of a former myocardial infarction. Rev Diabet Stud 2004, 1:175-184.

43. • Reyes-Soffer G, Holleran S, Karmally W, et al.: Measures of postprandial lipoproteins are not associated with coronary artery disease in patients with type $\mathbf{2}$ diabetes mellitus. $J$ Lipid Res 2009, 50:1901-1909. This study investigated the association between postprandial lipoproteins, including the size and number of TG-rich lipoproteins, and CVD risks among a relatively large number of diabetic patients.

44. Syvanne M, Talmud PJ, Humphries SE, et al.: Determinants of postprandial lipemia in men with coronary artery disease and low levels of HDL cholesterol. J Lipid Res 1997, 38:14631472.

45. Lopes-Virella MF, Klein RL, Virella G: Modification of lipoproteins in diabetes. Diabetes Metab Rev 1996, 12:69-90.

46. Bucala R, Makita Z, Koschinsky $T$, et al.: Lipid advanced glycosylation: pathway for lipid oxidation in vivo. Proc Natl Acad Sci U S A 1993, 90:6434-6438.

47. Hunt JV, Smith CC, Wolff SP: Autoxidative glycosylation and possible involvement of peroxides and free radicals in LDL modification by glucose. Diabetes 1990, 39:1420-1424.

48. Mullarkey CJ, Edelstein D, Brownlee M: Free radical generation by early glycation products: a mechanism for accelerated atherogenesis in diabetes. Biochem Biophys Res Commun 1990, 173:932-939.

49. Tentolouris N, Stylianou A, Lourida E, et al.: High postprandial triglyceridemia in patients with type 2 diabetes and microalbuminuria. J Lipid Res 2007, 48:218-225. 
50. Hirano T, Oi K, Sakai S, et al.: High prevalence of small dense LDL in diabetic nephropathy is not directly associated with kidney damage: a possible role of postprandial lipemia. Atherosclerosis 1998, 141:77-85.

51. Hayashi T, Hirano T, Taira T, et al.: Remarkable increase of apolipoprotein $B 48$ level in diabetic patients with end-stage renal disease. Atherosclerosis 2008, 197:154-158.
52. Yamamoto T, Hirano T, Mori Y, et al.: Significant increase of apolipoprotein $\mathrm{B} 48$ levels by a standard test meal in type 2 diabetic patients with nephropathy. J Atheroscler Thromb 2008, 15:199-205.

53. Turner RC, Millns H, Holman RR: Coronary heart disease and risk factors in NIDDM - experience from the United Kingdom Prospective Diabetes Study. Diabetologia 1997, 40(Suppl 2):S121-S122. 